\title{
Retraction Note to: The Crucial Role of Cyclin-Dependent Kinase-5-Ataxia-Telangiectasia Mutated Axis in ICH-Induced Neuronal Injury of Rat Model
}

\author{
Jing Wu ${ }^{1} \cdot$ Xin Zhang ${ }^{2} \cdot$ Yi Yan ${ }^{3} \cdot$ Zhaohua Tang $^{3} \cdot$ Xiaochuan Sun $^{3} \cdot$ Gang Huo $^{3}$. \\ Zhengbu Liao ${ }^{3}$
}

Published online: 1 July 2017

(C) Springer Science+Business Media, LLC 2017

Retraction Note to: Mol Neurobiol (2016) 53:6301-6308

DOI 10.1007/s12035-015-9524-4

This article has been retracted at the request of the Editorin-Chief and the Publisher per the Committee on Publication Ethics guidelines. There is a strong reason to believe that the peer review process was compromised. As such the validity of the content of this article cannot be verified.

The online version of the original article can be found at http://dx.doi.org/ $10.1007 / \mathrm{s} 12035-015-9524-4$

\section{Zhengbu Liao}

liaozhengbu163@163.com

1 Intensive Care Unit, The Second Affiliated Hospital of Chongqing Medical University, Chongqing 400010, China

2 Institute of Molecular Biology, Chongqing Medical University, Chongqing 400016, China

3 Department of Neurosurgery, The First Affiliated Hospital of Chongqing Medical University, Chongqing 400016, China 